Pacific Journal of Mathematics

UNIMODULAR SOLUTIONS OF INFINITE SYSTEMS OF 


\title{
UNIMODULAR SOLUTIONS OF INFINITE SYSTEMS OF LINEAR EQUATIONS
}

\author{
Donald C. Benson
}

It is well known that if a series of real numbers $\sum_{n=1}^{\infty} a_{n}$ converges, but not absolutely, then for any $b$, there exists a sequence $\left\{x_{i}\right\}, x_{i}= \pm 1$, such that $\sum_{n=1}^{\infty} a_{n} x_{n}=b$. In $\S \mathbf{1}$, a criterion is given on a system of denumerably many equations of this type, with real coefficients, so that solutions $x_{i}= \pm 1$ exist for arbitrary right hand sides. A sequence $\left\{x_{i}\right\}$ such that $x_{i}= \pm 1$ will be called unimodular. In $\S 2$, there results are extended to finite systems, and it is shown that an infinite system has unimodular solutions for arbitrary right hand sides if and only if every finite subsystem has this property. $\S 3$ shows that if a system satisfies the criterion of $\S 1$, then, in a certain sense, "almost any" sequence $\left\{x_{i}\right\}$, $x_{i}= \pm 1$, "satisfies" the system for any choice of right hand sides. In $\S 4$, conditions are given whereby infinite systems can be constructed which satisfy the criterion of $\S 2$. It follows, for example, that the system

$$
\sum_{j=1}^{\infty}(-1)^{\left[j / 2^{i}\right]} j^{-\alpha} x_{j}=b_{i}, \quad i=1,2, \cdots ; 0<\alpha \leqq 1
$$

has solutions $\left(x_{i}= \pm 1\right)$ for any $b_{i}(i=1,2, \cdots)$. The $b_{i}$ are allowed to be real numbers or $\pm \infty$.

1. The main theorem. Theorem 1. Let $a_{i j}(i, j=1,2,3, \cdots)$ be real numbers such that there exist $x_{j k l}(j=1,2, \cdots ; k=0,1,2, \cdots$; $l=1,2, \cdots)$ which satisfy the following conditions:

1. Each $x_{j k l}$ is equal to +1 or -1 .

2. $\sum_{j=1}^{\infty} a_{i j} x_{j k l}$ converges for all $i$ such that $i \neq k$ and $i \leqq l$.

3. $\sum_{j=1}^{\infty} a_{i j} x_{j_{\imath} l}$ diverges to $+\infty$.

Then, for any sequence $\left\{b_{i}\right\}$, the infinite system of equations

$$
\sum_{j=1}^{\infty} a_{i j} x_{j}=b_{i}
$$

can be solved such that for each $i, x_{i}= \pm 1$. Here, $b_{i}$ is allowed to be either a real number or $\pm \infty$.

Proof. If $k \neq i \leqq l$, for any $\varepsilon>0$ there exists $N(\varepsilon ; i, k, l)$ such

Received January 22, 1964. 
that

$$
\left|\sum_{j=m+1}^{n} a_{i j} x_{j k l}\right|<\varepsilon \text { and }\left|a_{i n}\right|<\varepsilon / 2,
$$

provided $m, n>N(\varepsilon ; i, k, l)$.

We define the solution $\left\{x_{i}\right\}$ inductively along with positive integers $M_{n m}$ which will be defined whenever $n$ is a positive integer and $m$ is a nonnegative integer such that $m \leqq n$. The ordered pairs $(n, m)$ are ordered lexicographically, i. e., $(n, m)<\left(n_{1}, m_{1}\right)$ if and only if either $n<n_{1}$, or $n=n_{1}$ and $m<m_{1}$. The induction will be with respect to this order.

The following definitions will be used with $m \leqq n$ and $i \leqq n$ :

$$
B_{i n m}=\left\{\begin{array}{l}
b_{i} \text { if } b_{i} \text { is finite } \\
\pm n \text { if } b_{i}= \pm \infty \text { and } i \leqq m, \\
\pm(n-1) \text { if } b_{i}= \pm \infty \text { and } i>m
\end{array}\right.
$$

$$
A_{i n m}=\left\{\begin{array}{l}
b_{i} \text { if } b_{i} \text { is finite } \\
B_{i n m} \text { if } i \neq m, \\
\pm(n-1) \text { if } i=m \text { and } b_{i}= \pm \infty
\end{array}\right.
$$$$
\delta_{i n m}=\left\{\begin{array}{l}
(2(m-i)+1) / n^{2} \text { if } i<m \\
2(n-i) /(n-1)^{2}+2 m / n^{2} \text { if } i \geqq m .
\end{array}\right.
$$

Let us suppose that positive integers $M_{n m}$ have been defined for $(n, m) \leqq(s, t)$, and $x_{i}$ for $i \leqq M_{s t}$ such that the following conditions are satisfied:

(A) $M_{n m}<M_{p q}$ if and only if $(n, m)<(p, q)$.

(B) $M_{n 0} \geqq N\left(1 / n^{2} ; i, k, n\right)$ for all $i, k \leqq n(i \neq k)$.

(C) $\left|\sum_{j=1}^{M_{n m}} a_{m j} x_{m}-B_{m n m}\right|<1 / n^{2}$ where $m \neq 0$.

(D) If $i \leqq n-1$ and $M_{n m} \geqq p>\left\{\begin{array}{l}M_{n, m-1} \text { if } m \neq 0 \\ M_{n-1, n-1} \text { if } m=0,\end{array}\right.$ then and

(D1) $A_{i n m}-\delta_{i n m}<\sum_{j=1}^{p} a_{i j} x_{j}<B_{i n m}+\delta_{i n m}$ if $A_{i n m} \leqq B_{i n m}$

(D2) $B_{i n m}-\delta_{i n m}<\sum_{j=1}^{p} a_{i j} x_{j}<A_{i n m}+\delta_{i n m}$ if $B_{i n m} \leqq A_{i n m}$.

We wish to determine $M_{u v}$ where $(u, v)$ is the immediate successor of $(s, t)$, and $x_{i}, i=M_{s t}+1, \cdots, M_{u v}$, such that the conditions (A)-(D) are valid for all $(n, m) \leqq(u, v)$. There are two cases to consider. Either we have $s=t$, or $s>t$. 
Case I. $s=t$. In this case the immediate successor of $(s, t)$ is $(s+1,0)$. Putting $M_{s+1,0}$ equal to the largest of the numbers $M_{s s}+1$ and $N\left(1 /(s+1)^{2} ; i, k, s+1\right)$ for all $i, k \leqq s+1(i \neq k)$, we see that (A) and (B) will be satisfied.

We now put $x_{j}=x_{j o s}, \quad\left(j=M_{s s}+1, \cdots, M_{s+1.0}\right)$. Condition (C) remains satisfied because the newly defined quantities do not occur in (C). Condition (B) holds with $n=s$ by the inductive assumption. Therefore, $\left|\sum_{j=k+1}^{l} a_{i j} x_{j o s}\right|<1 / s^{2}$, provided $k, l>M_{s s}$ and $i \leqq s$. We have $\delta_{i s s}=(2(s-i)+1) / s^{2}, \delta_{i, s+1,0}=2(s+1-i) / s^{2}$, and hence $\delta_{i, s+1,0}$ $-\delta_{i s s}=1 / s^{2}$. From the equality $A_{i s s}=B_{i s s}=A_{i, s+1,0}=B_{i, s+1,0}$ for $i<s$, we see that (D) holds with $i<s$. It must be shown that (D) holds with $i=s$. We have also $B_{s s s}=B_{s, s+1,0}=A_{s, s+1,0}$. Recall that

$$
\left|\sum_{j=M}^{l} a_{s s} a_{i j} x_{j o s}\right|<1 / s^{2}
$$

Since $\delta_{s, s+1,0}=2 / s^{2}$ and (C) holds with $i=s$, the result follows, namely that (D) holds with $i=s$. This disposes of Case I.

Case II. $s>t$. The immediate successor of $(s, t)$ is $(s, t+1)$. We use the fact that $\sum_{j=1}^{\infty} a_{t+1, j} x_{j, t+1, s}=+\infty$.

Subcase IIA. For some $l>M_{s t}$ we have

$$
\left|\sum_{j=1}^{l} a_{t+1, j} x_{j o s}-B_{t+1, s, t+1}\right|<1 / s^{2}
$$

In this case, we put $l=M_{s, t+1}$ and $x_{j}=x_{j o s}, j=M_{s t}+1, \cdots, M_{s, t+1}$.

Subcase IIB. If the above never happens, then

$$
\sum_{j=1}^{l} a_{t+1, j} x_{j o s}-B_{t+1, s, t+1}
$$

must keep the same sign for all $l>M_{s t}$, because, for $j>M_{s t}$, we have $\left|a_{t+1, j}\right|<1 / 2 s^{2}$, from inductive assumption (B).

Let $\sigma= \pm 1$, depending upon whether the sign stays + or - . Because the series $\sum_{j=1}^{\infty} a_{t \uparrow 1, j} x_{j, t+1, s}$ diverges to $+\infty$, there exists $K>M_{s t}$ such that

$$
\sum_{j=M_{s t}+1}^{l} a_{t+1, j} x_{j o s}+\sum_{j=M_{s t}+1}^{l} a_{t+1, j} x_{j, t+1, s}>0
$$

for all $l>K$. Let $K_{0}$ be the smallest number $K$ with this property. We put $x_{j}=x_{j o s}, M_{s t}<j<K_{0}$, and $x_{j}=-x_{j, t+1, s}, j=K_{0}, \cdots, M_{s, t+1}$, where the integer $M_{s, t+1}$ will now be defined. Because $\sum_{j=1}^{\infty} a_{t+1, j} x_{j, t+1, s}$ $=+\infty$, and $\left|a_{t+1, j}\right|<1 / 2 s^{2}$ for $j>M_{s t}$, there exist integers $M>M_{s t}$ 
such that (C) is satisfied for $n=s, m=t+1$ and $M_{s, t+1}=M$. Let $M_{s, t+1}$ be the smallest integer with the above property.

It will be shown that conditions (A)-(D) hold for both subcases. Conditions (A) and (B) are evident. Condition (C) follows immediately from the construction above. Condition (D) is somewhat more difficult.

It will be shown first that (D) holds with $i=t+1, n=s, m=$ $t+1$. We may suppose $i \leqq n-1$, i.e., $t+1 \leqq s-1$. We have

$$
\delta_{t+1, s, t+1}-\delta_{t+1, s, t}=2 / s^{2},
$$

and

$$
B_{t+1, s, t}=A_{t+1, s, t}=A_{t+1, s, t+1} .
$$

From inductive assumption (D), we have

$$
\left|\sum_{j=1}^{M_{s t}} a_{t+1, j} x_{j}-A_{t+1, s, t+1}\right|<\delta_{t+1, s, t+1}-2 / s^{2} .
$$

Thus, in Subcase IIA with $M_{s t}<p \leqq M_{s, t+1}$, and in Subcase IIB, with $M_{s t}<p<K_{0}<M_{s, t+1}$, we have

$$
\left|\sum_{j=1}^{p} a_{t+1, j} x_{j}-A_{t+1, s, t+1}\right|<\delta_{t+1, s, t+1}-1 / s^{2} .
$$

This disposes of Subcase IIA, because the above inequality is stronger than (D). It must be shown now that (D) holds for $K_{0} \leqq p \leqq M_{s, t+1}$, with $i=t+1, n=s, m=t+1$.

From the inequalities,

$$
\begin{aligned}
& \sigma \sum_{i-1, t, 1}^{K_{0}} a_{t+1, j} x_{j o s}+\sum_{i=1 . .}^{K_{0}} a_{t+1, j} x_{j, t+1, s}>0, \\
& J-\mu s t+1 \quad J=\mu_{s t}+1 \\
& \sigma \sum_{j=M_{s t}+1}^{K_{0}-1} a_{t+1, j} x_{j o s}+\sum_{j=M_{s t}+1}^{K_{0}-1} a_{t+1, j} x_{j, t+1, s} \leqq 0,
\end{aligned}
$$

(where an empty sum is taken to equal zero), it follows that we have. $\left(p \leqq M_{s, t+1}\right)$

$$
\begin{aligned}
\sum_{j=M_{s t}+1}^{K_{0}-1} a_{t+1, j} x_{j o s}- & \sum_{j=K_{0}}^{p} a_{t+1, j} x_{j, t+1, s} \\
& =\sigma \sum_{j=M_{s t}+1}^{p} a_{t+1, j} x_{j} \\
& <-\sum_{j=M_{s t+1}}^{p} a_{t+1, j} x_{j, t+1, s}+2\left|a_{t+1, K_{0}}\right| \\
& <-\sum_{j=M_{s t+1}}^{p} a_{t+1, j} x_{j, t+1, s}+1 / s^{2}
\end{aligned}
$$

using (B) and the definition of $x_{j}, M_{s t}<j \leqq M_{s, t+1}$. Now, from (6), we have 


$$
-\sum_{j=M_{s t+1}}^{p} a_{t+1, j} x_{j, t+1, s}<-\sigma \sum_{j=M_{s t}+1}^{p} a_{t+1, j} x_{j o s} .
$$

Combining (1), (11) and (B), we have

$$
\sigma \sum_{j=M_{s t}+1}^{p} a_{t+1, j} x_{j}<-\sigma \sum_{j=M_{s t}+1}^{p} a_{t+1, j} x_{j o s}+1 / s^{2}<2 / s^{2} .
$$

From (8), we have

$$
\sigma \sum_{j=1}^{M s t} a_{t+1, j} x_{j}<\sigma A_{t+1, s, t+1}+\delta_{t+1, s, t+1}-1 / s^{2} .
$$

Putting (12) and (13) together, we have

$$
\sigma \sum_{\jmath=1}^{p} a_{t+1, j} x_{j}<\sigma A_{t+1, s, t+1}+\delta_{t+1, s, t+1} .
$$

From the definition of $\sigma$ and the fact that if $A_{t+1, s, t+1} \neq B_{t+1, s, t+1}$, then $\left|A_{t+1, s, t+1}-B_{t+1, s, t+1}\right|=1$, it follows that $\sigma\left(A_{t+1, s, t+1}-B_{t+1, s, t+1}\right) \geqq 0$. Thus (D1) or (D2) must be demonstrated, depending on whether $\sigma=$ -1 or +1 .

If $\sigma=-1$, we have from (14)

$$
A_{t+1, s, t+1}-\delta_{t+1, s, t+1}<\sum_{j=1}^{p} a_{t+1, j} x_{j}
$$

which is half of (D1). From the definition of $M_{s, t+1}$, we have

$$
\sum_{j=1} a_{t+1, j} x_{j}<B_{t+1, s, t+1}+1 / s^{2}<B_{t+1, s, t+1}+\delta_{t+1, s, t+1},
$$

provided $M_{s t}<p \leqq M_{s, t+1}$, which gives the remaining half of (D1). Similar considerations show that if $\sigma=1$, then (D2) holds. This concludes the demonstration that (D) holds with $i=t+1, n=s, m=$ $t+1$.

It remains to show that (D) holds for $i \neq t+1, n=s, m=t+1$, $i \leqq s-1$.

We have $\delta_{i, s, t+1}-\delta_{i, s, t}=2 / s^{2}$, so that it is sufficient to show

$$
\left|\sum_{j=M s t}^{p} a_{i j} x_{j}\right|<2 / s^{2}
$$

for $M_{s t}<p \leqq M_{s, t+1}$.

In Subcase IIA, we have, using (B),

$$
\left|\sum_{j=M s t+1}^{p} a_{i j} x_{j}\right|=\left|\sum_{j=M M_{s t}+1}^{p} a_{i j} x_{j o s}\right|<1 / s^{2} .
$$

In Subcase IIB with $p<K_{0}$, (15) is valid once again because (16) holds. 
In Subcase IIB with $p \geqq K_{0}$, we have

$$
\begin{aligned}
\left|\sum_{j=M_{s t}+1}^{p} a_{i j} x_{j}\right| \leqq & \left|\sum_{j=M_{s t}+1}^{K_{0}-1} a_{i j} x_{j o s}\right| \\
& +\left|\sum_{j=K_{0}}^{p} a_{i j} x_{j, t+1, s}\right|<\left(1 / s^{2}\right)+\left(1 / s^{2}\right)
\end{aligned}
$$

because of (B). This concludes the demonstration of Case II.

The definition by induction is completed by setting $M_{01}=N(1 ; 1,0,1)$ and observing that thereby (A)-(D) are satisfied with $n=1, m=0, i=1$.

It remains to show the sequence $\left\{x_{i}\right\}$ constructed in this way satisfies the infinite system of equations (1). However, this follows from the fact that $\left\{x_{i}\right\}$ satisfies condition (D).

2. Systems with finitely many equations. The extension of Theorem 1 to systems with finitely many equations is accomplished by producing an infinite system which can be treated by Theorem 1 and which is equivalent to the given finite system.

THEOREM 2. Let $a_{i j}(i=1, \cdots, R ; j=1,2, \cdots)$ be real numbers such that there exist $x_{j k}(j=1,2, \cdots ; k=0,1, \cdots, R)$ which satisfy the following conditions:

1. Each $x_{j k}$ is equal to +1 or -1 .

2. $\sum_{j=1}^{\infty} a_{i j} x_{j k}$ converges for all $i$ such that $i \neq k$.

3. $\sum_{j=1}^{\infty} a_{i j} x_{j i}$ diverges to $+\infty$.

Then, for any numbers $b_{1}, \cdots, b_{R}$, each of which is a real number or $\pm \infty$, the equations

$$
\sum_{j=1}^{\infty} a_{i j} x_{j}=b_{i}, \quad(i=1, \cdots, R)
$$

can be solved such that for each $i, x_{i}= \pm 1$.

Proof. We construct an auxiliary infinite system of equations

$$
\sum_{j=1}^{\infty} \alpha_{i j} \xi_{j}=\beta_{i} \quad(i=1,2, \cdots) .
$$

We define $\beta_{i+n R}=b_{i}$ for any nonnegative integer $n$, and

$$
\alpha_{i+n R, l}=\left\{\begin{array}{l}
a_{i k} \text { if there exists } k>0 \text { such that } \\
l=T(k+n-1)+n-1 \text { and } \\
0 \text { otherwise, }
\end{array}\right.
$$

where $T(n)=n(n+1) / 2$ is the $n$th triangular number. The fact to 
be used about $T(n)$ is that each positive integer has one and only one representation in the form $T(k+n-1)+n-1=S(k, n)$, where $k$ and $n$ are positive integers.

Let us define $\xi_{j k l}=\xi_{j k}$ as follows:

(21) $\xi_{l, i+n R}=\left\{\begin{array}{l}x_{k i} \text { if there exists } k>0 \text { such that } l=S(k, n) \text {, and } \\ x_{k 0} \text { if } l=S(k, m), n \neq m>0 .\end{array}\right.$

Then we have

1. Each $\xi_{j k}$ is equal to +1 or -1 .

2. $\sum_{j=1}^{\infty} \alpha_{i j} \xi_{j k}$ converges for all $i$ such that $i \neq k$.

3. $\sum_{j=1}^{\infty} \alpha_{i j} \xi_{j i}$ diverges to $+\infty$.

The hypotheses of Theorem 1 are satisfied, and therefore the system (19) has a solution $\left\{\xi_{j}\right\}$. Then $x_{j}=\xi_{T(j)}, j=1,2, \cdots$, is a solution of (18).

COROLLARY. The system (1), with arbitrary right hand sides, has a unimodular solution if and only if every finite subsystem of (1), with arbitrary right hand sides has a unimodular solution.

A system of nondenumerably many equations of the type described in Theorem 1 will never have unimodular solutions for all possible right hand sides, because the number of ways in which the right hand sides could be prescribed would have cardinality greater than $C$, whereas the cardinality of all unimodular sequences, $x_{i}= \pm 1, i=1,2, \cdots$, is equal to $C$. (Here $C$ denotes the cardinality of the continuum.)

3. The metric space $\mathscr{l}$. The set of sequences $\left\{x_{i}\right\}, x_{i}= \pm 1$ form a complete metric space under the metric

$$
d\left(\left\{x_{i}\right\},\left\{x_{i}^{\prime}\right\}\right)=1 / l,
$$

where $l=\min \left\{i: x_{i} \neq x_{i}^{\prime}\right\}$.

Let $\mathrm{a}_{i j}$ satisfy the hypotheses of Theorem 1 . Let $U_{i}, i=1,2, \cdots$, be nonempty open sets of extended real numbers. ( $U_{i}$ may contain $+\infty$ or $-\infty$.) Let $\mathscr{N}_{M}$ be the set of sequences $\left\{x_{i}\right\}$ such that for all $N \geqq M, \sum_{j=1}^{N} a_{i j} x_{j} \notin U_{i}$ for some $i(0<i \leqq M)$.

$\mathscr{N}_{M}$ is closed. For suppose $\left\{x_{i}^{n}\right\} \in \mathscr{N}_{M}$ and $\lim _{n \rightarrow \infty} d\left(\left\{x_{i}^{n}\right\},\left\{x_{i}\right\}\right)=0$. Also, suppose there exists $N \geqq M$ such that $\sum_{j=1}^{N} a_{i j} x_{j} \in U_{i}$ for each $i(0<i \leqq M)$. For sufficiently large $n$, we have $x_{j}=x_{j}^{n}, j=1, \cdots, N$, and hence we get $\sum_{j=1}^{N} a_{i j} x_{j}^{n} \in U_{i}(0<i \leqq M)$, contrary to the assumption $\left\{x_{i}^{n}\right\} \in \mathscr{N}_{M}$.

$\mathscr{N}_{M}$ is nowhere dense. For suppose $\left\{x_{i}\right\} \in \mathscr{N}_{M}$. Let $b_{i}$ be an 
arbitrary element of $U_{i}$. For any $P>0$, there exists, because of Theorem 1 , a sequence $\left\{x_{i}\right\}$ such that

1. $x_{i}^{\prime}=x_{i}, i=1,2, \cdots, P$, and

2. $\sum_{j=1}^{\infty} a_{i j} x_{j}^{\prime}=b_{i}$.

Clearly, $\left\{x_{i}^{\prime}\right\} \notin \mathscr{N}_{M}$ and $d\left(\left\{x_{i}\right\},\left\{x_{i}^{\prime}\right\}\right)<1 / P$.

Thus the set $\bigcup_{M=1}^{\infty} \mathscr{N}_{M}=\mathscr{N}$ is of the first category, and since $\mathscr{C l}$ is a complete metric space, $\mathscr{C l}-\mathscr{N}$ is of the second category. We have proved the following:

Lemma 1. For any sequence $\left\{x_{n}\right\}$ in $\mathscr{C l}-\mathscr{N}$ there exists an infinite monotone increasing sequence $\left\{N_{k}\right\}$ of positive integers such that for each $k, \sum_{j=1}^{N k} a_{i j} x_{j} \in U_{i}$ for $i \leqq k$.

For any sequence $\left\{b_{i}\right\}$ of extended real numbers we may take $U_{i}^{n}$ as follows:

$$
U_{i}^{n}=\left\{\begin{array}{l}
\left\{x:\left|x-b_{i}\right|<1 / n\right\} \text { if } b_{i} \text { is finite } \\
\left\{x: \pm\left(x-b_{i}\right)>n\right\} \text { if } b_{i}= \pm \infty
\end{array}\right.
$$

By applying the lemma for each $n$ to $\left\{U_{i}^{n}\right\} 0<i<\infty$, we find that there exists a monotone increasing sequence of positive integers $\left\{S_{k}\right\}$ such that

$$
\sum_{j=1}^{s_{k}} a_{i j} x_{j} \in U_{i}^{k} \quad \text { for } i \leqq k
$$

From (22), it now follows that we have

$$
\lim _{k \rightarrow \infty} \sum_{j=1}^{s_{k}} a_{i j} x_{j}=b_{i} \quad \text { for every } i>0 \text {. }
$$

In summary, this proves the following:

THEOREM 3. Let $a_{i j}$ satisfy the hypotheses of Theorem 1. Then there exists a sequence $\left\{x_{i}\right\}, x_{i}= \pm 1$, with the following property. (Indeed, any sequence $\left\{x_{i}\right\}$ in the complete metric space $\mathscr{C l}$, apart from a certain set of first category, has this property.) For any sequence $\left\{b_{i}\right\}$ of extended real numbers, there exists a sequence of positive integers $\left\{S_{k}\right\}$ such that for each $i$,

$$
\lim _{k \rightarrow \infty} \sum_{j=1}^{s_{k}} a_{i j} x_{j}=b_{i} .
$$

4. Sufficient conditions. In this section we shall find sufficient conditions on the coefficients $a_{i j}$ so that the hypotheses of Theorem 1 are satisfied. 
THEOREM 4. Let $\left\{a_{i}\right\}$ be a sequence of real numbers such that

(i) $a_{i}>0$

(ii) $\sum_{i=1}^{\infty} a_{i}=\infty$.

(iii) For every $k \geqq 1, a_{i}-a_{j+k}$ is monotone decreasing in $i$.

(iv) $a_{i}$ tends monotonically to zero as $i \rightarrow \infty$. Let $a_{i j}=(-1)^{[j / 2 i]} a_{j}$. Then $a_{i j}$ satisfies the hypotheses of Theorem 1 .

Proof. We must find sequences $\left\{x_{j k l}\right\}, 1 \leqq j<\infty, 0 \leqq k<\infty, 1 \leqq$ $l<\infty$, such that conditions (1), (2) and (3) of Theorem 1 are fulfilled.

$k=0$. First we show that by putting $x_{j 0 l}=1$, the conditions are satisfied for $k=0$. Condition (3) is fulfilled vacuously and condition (1) is trivial.

It will be shown that condition (2) holds, i.e., that $\sum_{j=1}^{\infty}(-1)^{[j / 2 i]} a_{j}$ converges for each $i$. Let

$$
(-1)^{k} b_{k}=\sum_{j=k \cdot 2^{i}}^{(k+1) 2^{i}-1}(-1)^{\left[j / 2^{i}\right]} a_{j} .
$$

Then we have

$$
b_{k}=\sum_{j=k \cdot 2^{i}}^{(k+1) i^{i}-1} a_{j}>0 .
$$

From (iv), $b_{k}$ is monotone decreasing, and hence $\sum(-1)^{k} b_{k}$ converges. The condition (2) follows because

$$
\sum_{j=1}^{k}(-1)^{i} b_{j}=\sum_{j=0}^{(k+1) 2^{i}-1}(-1)^{[j / 2 i]} a_{j} .
$$

$k \neq 0$. Let $x_{j k l}=(-1)^{\left[j / 2^{k}\right]}$. Since it is assumed that $\sum_{i=1}^{\infty} a_{i}=\infty$, we have $\sum_{j=1}^{\infty} a_{i j} x_{j i l}=\infty$, and thus condition (3) holds.

We will show (2) holds and thereby complete the proof by showing that

$$
\sum_{j=1}^{\infty}(-1)^{\left[j / 2^{i}\right]+[j / 2 k]} a_{j}
$$

converges if $i>k$. We have

$$
\left[j / 2^{i}\right]+\left[j / 2^{k}\right]=\left[\left(j+2^{i}\right) / 2^{i}\right]+\left[\left(j+2^{i}\right) / 2^{i}\right]-1-2^{i-k}
$$

and

$$
(-1)^{\left[j / 2^{i}\right]+[j / 2 k]}=-(-1)^{\left[\left(j+2^{i}\right) / 2^{i}\right]+\left[\left(j+2^{i}\right) / 2^{k} k_{j}\right.} .
$$

Putting

$$
(-1)^{n} c_{n}=\sum_{j=n \cdot 2^{i}}^{(n+1) 2^{i}-1}(-1)^{\left[j / 2^{i}\right]+\left[j / 2^{k}\right]} a_{j},
$$


we have

$$
\begin{aligned}
c_{n} & =\sum_{j=n \cdot 2^{i}}^{(n+1) 2^{i}-1}(-1)^{\left[j / 2^{k}\right]} a_{j} \\
& =\sum_{j=n \cdot 2^{i}} \sum_{m=0}^{n \cdot 2^{i} 2^{k}-1}\left(a_{j+2 m \cdot 2 k}-a_{j+(2 m+1) \cdot 2 k}\right) .
\end{aligned}
$$

Evidently $c_{n}$ is positive. Also, $c_{n}$ is monotone because, from (iii), $a_{i}-a_{i+2^{k}}$ is monotone decreasing in $i$. Thus $\sum_{n=1}^{\infty}(-1)^{n} c_{n}$ converges. Since we have

$$
\sum_{j=1}^{n}(-1)^{j} c_{j}=\sum_{j=0}^{n \cdot 2^{i}-1}(-1)^{\left[j / 2^{i}\right]+\left[j / 2^{k}\right]} a_{j}
$$

it follows that (27) converges if $i>k$. This concludes the proof of Theorem 4.

The sequence $a_{i}=1 / i^{\alpha}$, for positive $\alpha \leqq 1$, is an example satisfying (i)-(iv) of Theorem 4.

This result can be extended with the help of Abel's test for convergence.

THEOREM 5. Let $\left\{a_{i}\right\}$ satisfy the hypotheses of Theorem 4. Let $\left\{v_{i j}\right\}, i, j=1,2, \cdots$ satisfy the following:

1. $v_{i j}>0$.

2. For each $i,\left\{v_{i j}\right\}$ is monotone (increasing or decreasing) with respect to $j$.

3. $\sum_{j=1}^{\infty} a_{j} v_{i j}=\infty$ for each $i$.

Then $(-1)^{\left[j / 2^{i}\right]} a_{j} v_{i j}$ satisfies the hypotheses of Theorem 1 .

Proof. We take the same definition for $x_{j k}$ as in Theorem 4. Then $\sum_{j=1}^{\infty} a_{i j} x_{j k}$ converges for $i \neq k$ by Abel's test. Further, we have

$$
\sum_{j=1}^{\infty} a_{i j} x_{j i l}=\sum_{i=1}^{\infty} a_{j} v_{j i}=+\infty
$$

We obtain a result which allows us to transform any array of coefficients $a_{i j}$ which satisfies the hypotheses of Theorem 1 into a different array satisfying the same conditions. First we need a lemma which is related to Abel's test for convergence.

LEMMA 2. Let $\left\{v_{i}\right\}$ be a monotone decreasing sequence of real numbers which is bounded away from zero; i.e., there exists $b$ such that $0<b \leqq v_{i}$ for all $i$. Suppose $\sum_{i=1}^{\infty} a_{i}=+\infty$. Then $\sum_{i=1}^{\infty} v_{i} a_{i}=$ $+\infty$. 
Proof. Let $s_{n}=\sum_{i=1}^{n} a_{i}$, and let $h_{n}=\inf _{k \geqq n} s_{k}$. We have, if $m \leqq p$,

$$
\begin{aligned}
\sum_{i=1}^{p} a_{i} v_{i} & =s_{1}\left(v_{1}-v_{2}\right)+\cdots+s_{p-1}\left(v_{p-1}-v_{p}\right)+s_{p} v_{p} \\
& \geqq h_{1}\left(v_{1}-v_{m}\right)+h_{m} v_{m} \\
& \geqq-\left|h_{1}\right| v_{1}+h_{m} b .
\end{aligned}
$$

The result follows because $h_{m} \rightarrow \infty$ as $m \rightarrow \infty$.

THeorem 6. Let $a_{i j}$ satisfy the hypotheses of Theorem 1. Let $v_{i j}$ satisfy the following:

1. There exist $c_{i}$ such that $0<c_{i} \leqq v_{i j}$ for all positive integers $i$ and $j$.

2. For each $i,\left\{v_{i j}\right\}$ is monotone decreasing with respect to $j$.

Then $a_{i j} v_{i j}$ satisfy the hypotheses of Theorem 1.

Proof. The conditions are satisfied by using the $x_{j k l}$ which are assumed to exist in Theorem 1 . We have that $\sum_{j=1}^{\infty} a_{i j} v_{i j} x_{j k l}$ converges if $i \neq k$ by Abel's test and diverges to $+\infty$ for $i=k$ by Lemma 2 . University of CALIFornia, Davis 



\section{PACIFIC JOURNAL OF MATHEMATICS}

\section{EDITORS}

H. Samelson

Stanford University

Stanford, California

R. M. Blumenthal

University of Washington

Seattle, Washington 98105
J. Dugundu

University of Southern California Los Angeles, California 90007

*Richard Arens

University of California

Los Angeles, California 90024

\section{ASSOCIATE EDITORS}
E. F. BECKENBACH
B. H. NeumanN
F. WOLF
K. YOSIDA

\section{SUPPORTING INSTITUTIONS}

UNIVERSITY OF BRITISH COLUMBIA CALIFORNIA INSTITUTE OF TECHNOLOGY UNIVERSITY OF CALIFORNIA MONTANA STATE UNIVERSITY

UNIVERSITY OF NEVADA

NEW MEXICO STATE UNIVERSITY

OREGON STATE UNIVERSITY

UNIVERSITY OF OREGON

OSAKA UNIVERSITY

UNIVERSITY OF SOUTHERN CALIFORNIA
STANFORD UNIVERSITY

UNIVERSITY OF TOKYO

UNIVERSITY OF UTAH

WASHINGTON STATE UNIVERSITY

UNIVERSITY OF WASHINGTON

AMERICAN MATHEMATICAL SOCIETY CALIFORNIA RESEARCH CORPORATION SPACE TECHNOLOGY LABORATORIES NAVAL ORDNANCE TEST STATION 


\section{Pacific Journal of Mathematics}

\section{Vol. 15, No. $1 \quad$ September, 1965}

Donald Charles Benson, Unimodular solutions of infinite systems of linear

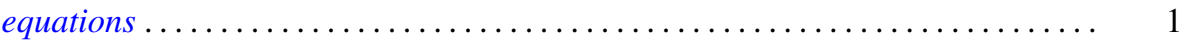

Richard Earl Block, Transitive groups of collineations on certain designs . . . . . . 13

Barry William Boehm, Existence of best rational Tchebycheff approximations .... . 19

Joseph Patrick Brannen, A note on Hausdorff's summation methods . . . . . . . . . . 29

Dennison Robert Brown, Topological semilattices on the two-cell ............ 35

Peter Southcott Bullen, Some inequalities for symmetric means . . . . . . . . . . 47

David Geoffrey Cantor, On arithmetic properties of coefficients of rational

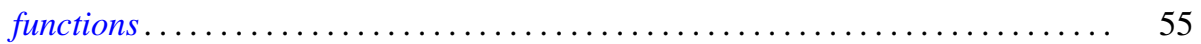

Luther Elic Claborn, Dedekind domains and rings of quotients . . . . . . . . . 59

Allan Clark, Homotopy commutativity and the Moore spectral sequence ........ 65

Allen Devinatz, The asymptotic nature of the solutions of certain linear systems of

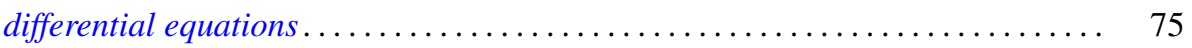

Robert E. Edwards, Approximation by convolutions ................... 85

Theodore William Gamelin, Decomposition theorems for Fredholm operators . . . . . 97

Edmond E. Granirer, On the invariant mean on topological semigroups and on

topological groups .................................. 107

Noel Justin Hicks, Closed vector fields . . . . . . . . . . . . . . . . . . . 141

Charles Ray Hobby and Ronald Pyke, Doubly stochastic operators obtained from

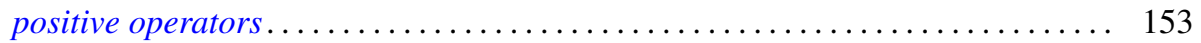

Robert Franklin Jolly, Concerning periodic subadditive functions . . . . . . . . . 159

Tosio Kato, Wave operators and unitary equivalence . . . . . . . . . . . . . . 171

Paul Katz and Ernst Gabor Straus, Infinite sums in algebraic structures . . . . . . . 181

Herbert Frederick Kreimer, Jr., On an extension of the Picard-Vessiot theory ...... 191

Radha Govinda Laha and Eugene Lukacs, On a linear form whose distribution is

identical with that of a monomial ......................... 207

Donald A. Ludwig, Singularities of superpositions of distributions . . . . . . . . . 215

Albert W. Marshall and Ingram Olkin, Norms and inequalities for condition

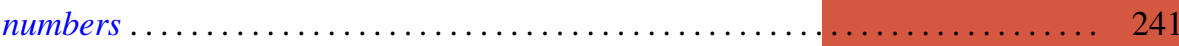

Horace Yomishi Mochizuki, Finitistic global dimension for rings . . . . . . . . . . 249

Robert Harvey Oehmke and Reuben Sandler, The collineation groups of division

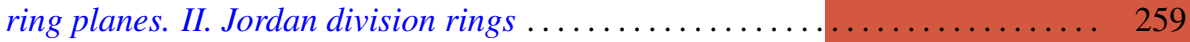

George H. Orland, On non-convex polyhedral surfaces in $E^{3} \ldots \ldots \ldots \ldots \ldots \ldots \ldots 267$

Theodore G. Ostrom, Collineation groups of semi-translation planes . . . . . . . . 273

Arthur Argyle Sagle, On anti-commutative algebras and general Lie triple

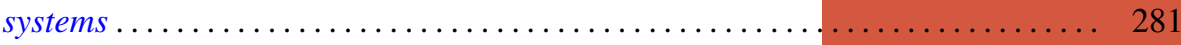

Laurent Siebenmann, A characterization of free projective planes . . . . . . . . . 293

Edward Silverman, Simple areas.................................. 299

James McLean Sloss, Chebyshev approximation to zero .................. 305

Robert S. Strichartz, Isometric isomorphisms of measure algebras . . . . . . . . . 315

Richard Joseph Turyn, Character sums and difference sets . . . . . . . . . . . . 319

L. E. Ward, Concerning Koch's theorem on the existence of arcs . . . . . . . . . . 347

Israel Zuckerman, A new measure of a partial differential field extension ......... 357 